\title{
Fuzzy linear programming: an application to hydroelectric generation scheduling
}

\author{
R.-H. Liang \\ Y.-Y. Hsu
}

Indexing terms: Fuzzy linear programming, Fuzzy sets, Hydroelectric generation scheduling

\begin{abstract}
A new approach using fuzzy linear programming is proposed for solving the hydroelectric generation scheduling problem. A characteristic feature of this approach is that the errors in the forecast hourly loads and natural inflows can be taken into account by using fuzzy set notation, making the approach superior to the conventional linear programming method in which the hourly loads and natural inflows are assumed to be exactly known and there are no errors in the forecast loads and natural inflows. To reach an optimal schedule under the uncertain environment, a fuzzy linear programming model in which the hourly loads, the hourly natural inflows and the cost are all expressed in fuzzy set notations is developed. The developed fuzzy linear programming approach is applied to schedule the generation in the Taiwan power system which contains ten hydroplants including cascaded ones. It is found that the proposed approach is very effective in obtaining proper hydrogeneration schedules in uncertain conditions.
\end{abstract}

\section{Introduction}

The major objective of hydrogeneration scheduling in a power system is to minimise the total fuel cost of thermal units by utilising the limited water resource. It is a typical optimisation problem in which the total operating cost over the study period is minimised subject to load and all system constraints. Numerous approaches have been proposed for solving hydroelectric generation scheduling problem [1-8].

In conventional approaches to the hydroelectric generation scheduling problem, the power generation-load balance and the water balance equation must be maintained at each hour over the study period. In other words, the following equations must be satisfied at each hour $t$.

$$
\begin{aligned}
& \sum_{i \in M_{i}} P_{i}\left(X_{i t}\right)+G T H E R M A L_{t}=L_{t} \\
& Y_{i t+1}=Y_{i t}+\sum_{j \in N_{i}} X_{j t}-X_{i t}+R_{i t}
\end{aligned}
$$

(C) IEE, 1994

Paper 1357C (P10), first received 6th December 1993 and in revised form 10th May 1994

The authors are with the Department of Electrical Engineering, National Taiwan University, Taipei, Taiwan, Republic of China where

$$
\begin{aligned}
L_{t}= & \text { total system load at hour } t \\
Y_{i t}= & \text { water volume of reservoir } i \text { at begin- } \\
& \text { ning of hour } t \\
X_{i t}= & \text { volume of water released from } \\
& \text { reservoir } i \text { for generation during } \\
& \text { hour } t \\
P_{i}(\cdot)= & \text { water-to-energy conversion function } \\
& \text { of power plant associated with } \\
& \text { reservoir } i \\
M_{i}= & \text { set of reservoirs under study } \\
R_{i t}= & \text { volume of natural inflow to reservoir } \\
& i \text { during hour } t \\
G T H E R M A L_{t}= & \text { total power from thermal units at } \\
N_{i}= & \text { hour } t \\
& \text { of reservoir } i
\end{aligned}
$$

Note that the study period is one day $(24 \mathrm{~h})$ in the present work. It is also worth noting that eqn. 1 holds since transmission losses are normally neglected in the hydroelectric generation scheduling problem.

Eqns. 1 and 2 reveal that system load demand and natural inflows must be known before the hydroelectric generation scheduling problem can be tackled. Load demands and natural inflows can only be known through short-term forecasting. Since load demand and natural inflows depend on the social behavour of customers, weather variables, etc. there are always errors in the forecast system loads and natural inflows. This raises the equation of how to tackle the hydroelectric generation scheduling problem when the load demand and natural inflows are imprecise.

In this paper, the hydroelectric generation scheduling problem is formulated as a constrained optimisation problem. We use an aggregated thermal unit, which is obtained through dispatching available thermal units by the lambda-iteration method [9], to construct the thermal generation cost function and to linearise this function in the nearby operating point at every hour. It is assumed that the company requirements to produce at minimal cost, to meet the load demand and the water balance equation are not as crisp as is commonly believed. An approach based on fuzzy sets is proposed to reach the desired hydro generation schedules based on uncertain load demands and natural inflows.

Fuzzy models $[10,11]$ have received much attention from power engineers in the past few years. Many interesting applications of fuzzy sets in the power field have been reported [12-18]. There are several distinct formulations of fuzzy linear programming. The max-min formulation which has been widely employed by power engineers $[16,17]$ is adopted herein. Fuzzy sets $[10]$ were

IEE Proc.-Gener. Transm. Distrib., Vol. 141, No. 6, November 1994 
introduced by Zadeh for handling nonprobabilistic uncertainties associated with the structure of a set of objects. The fuzzy set theory is considered as a generalisation of traditional probability set theory. The idea is to replace the concept that each variable has a precise value by the fuzzy concept that each variable is assigned a degree of membership for each possible value of the variable. To reach an optimal generation schedule under the fuzzy environment, generation costs, load demand and natural inflows are all expressed in fuzzy set notation. Fuzzy linear programming [11] is then used to obtain the desired generation schedule.

The effectiveness of this technique has been demonstrated by hydrogeneration scheduling of the Taiwan power system which consists of four Ta-Chia River cascaded plants, three Cho-Shui River plants (including a large pumped storage plant and two cascaded hydroplants) and three hydraulically independent plants.

\section{The hydroelectric generation scheduling} problem

Scheduling hydrogeneration is well known to be coupled with its thermal counterpart. We now decouple the hydroscheduling from the thermal part by first assuming a purely thermal system. For each given load level, the lambda-iteration method is performed to solve the economic dispatch over the set of available units [9] and to evaluate the thermal generation cost to meet the load demand. In other words, we aggregate all the available thermal units into one equivalent unit and construct its generation cost function. Then, in hydroscheduling, we try to find the best way of substituting hydro for thermal energy based on this function so that the system generation cost is minimised. In the Taiwan Power Company's hydrosystem, there is no significant delay relative to the one-hour time increment for water to flow from one reservoir to its immediate downstream neighbour. To do this, the study period (one day for the present work) is divided into $N$ stages $(N=24$ in the present case) and the hydroscheduling problem is then formulated as follows:

$$
\text { minimise } C=\sum_{t=1}^{24} \operatorname{COST}\left(G T H E R M A L_{t}\right)
$$

subject to

(i) The generation-load balance equations

$$
\text { GTHERMAL } L_{t}+\sum_{i} P_{i}\left(X_{i t}\right)=L_{t} \quad t=1,2, \ldots, 24
$$

(ii) The water balance equations

$$
\begin{array}{r}
Y_{i t+1}=Y_{i t}+\sum_{j \in N_{i}} X_{j t}-X_{i t}+\sum_{i \in N_{i}} S_{i t}-S_{i t}+R_{i t} \\
i=1,2, \ldots, 10 \quad t=1,2, \ldots, 24
\end{array}
$$

(iii) Bounds on water releases

$\underline{X_{i}} \leqslant X_{i t} \leqslant \overline{X_{i}}$ and $\underline{S_{i}} \leqslant S_{i t} \leqslant \bar{S}_{i} \quad i=1,2, \ldots, 10$

(iv) Bounds on a reservoir storage

$\underline{Y}_{i} \leqslant Y_{i t} \leqslant \bar{Y}_{i} \quad i=1,2, \ldots, 10$

where

$C=$ system generation cost over study period

$\cos T_{t}(\cdot)=$ generation cost function at hour $t$ which is approximated by a second-order polynomial

$S_{i t}=$ spillage from reservoir $i$ during hour $t$
Note that the above formulation turns out to be a discrete time optimisation problem with linear constraints on both decision variables $X \mathbf{s}$ and state $Y$ s.

To deal with the optimisation problem, both dynamic programming and linear programming can be employed. In the present work, the linear programming approach is used to tackle the hydroscheduling problem. Since the convex objective function in eqn. 3 is a nonlinear function of the variable GTHERMAL, it is necessary to linearise this function first in the linear programming formulation. Fig. 1 depicts a piecewise linear approx-

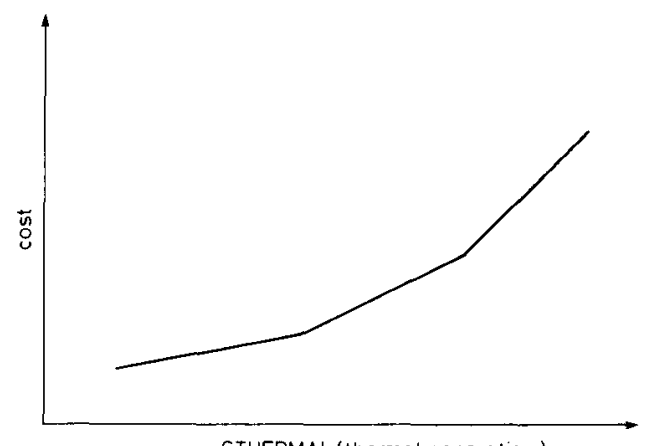

GTHERMAL (thermal generation)

Fig. 1 Thermal generation cost function approximated by multisegment piecewise linear curve

imation to the cost curve which is originally represented by a second-order polynomial.

The hydroscheduling problem can now be formulated as a general linear programming (LP) problem.

$$
\min _{x} C=\underline{C}^{t} \underline{X}
$$

subject to

$$
A \underline{X} \geqslant \underline{b}
$$

$$
\underline{X} \geqslant \underline{0}
$$

where

$C=$ total cost to be minimised

$X^{t}=\left[X_{1}, \ldots, X_{n}\right]=$ vector of control variables

$\underline{\boldsymbol{C}}^{t}=\left[c_{1}, \ldots, c_{n}\right]=$ vector of cost parameters

$\underline{b}^{t}=\left[b_{1}, \ldots, b_{m}\right]=$ constraint vector

$A=\left[a_{i j}\right]=$ constraint matrix

In conventional linear programming formulation, the elements of $A, \underline{b}$ and $C$ are all crisp numbers. The crisp inequality constraints can be replaced by fuzzy sets and the objective function can also be replaced by a fuzzy set. In this case, the fuzzy linear programming formulation to be described in the following section must be used instead of the conventional linear programming formulation.

\section{Proposed fuzzy linear programming approach}

Fuzzy set theory is a generalisation of traditional crisp set theory. As the underlying formulation of any optimisation problem relies on the set structure, optimisation problems under an uncertain environment can be reformulated using fuzzy sets. Some important definitions of fuzzy sets can be found in Reference 14.

In this section, a fuzzy linear programming model is developed based on fuzzy constraints and cost function. 
Fuzzy set formulation is used because we want a good solution to a model that represents the uncertainties inherent in a practical optimisation problem.

In the proposed fuzzy linear programming (FLP) approach, the power generation-load balance equation and water balance equation in eqns. 4 and 5 are treated as fuzzy constraints since they are related to the imprecise (fuzzy) hourly loads $L$ and natural inflows $R$. A fuzzy objective function characterised by a fuzzy set $\mathcal{C}$ related to total generation cost $C$ of the thermal units is used. Since the objective of hydroscheduling problem is to minimise the total generation cost, we can define a membership function for the fuzzy set $\tilde{C}$ so that a high cost is given a low membership value. By keeping the membership function as high as possible, a desirable solution with low generation cost can be reached.

The hydroscheduling problem originally formulated in eqns. 8-10 under crisp conditions must now be reformulated under fuzzy environments.

$\min \tilde{C}$

subject to

$A \underline{X} \geqslant \underline{b}$

$\underline{X} \geqslant \underline{0}$

where $\bar{C}$ in eqn. 11 is a fuzzy objective function and eqn. 12 is a fuzzy constraint.

In the proposed approach, the fuzzy objective in eqn 11 is reformulated as a fuzzy constraint. In other words, we want to keep the total cost below some maximum level of expense, $C^{M}$. Below this level of expense, a higher membership value indicates a better solution for that objective. Thus, eqns. 11-13 are reformulated as follows. Find $\underline{X}$ such that

$$
\begin{aligned}
& B X \geqslant \underline{d} \\
& \underline{X} \geqslant \underline{0}
\end{aligned}
$$

where

$$
B=\left(\begin{array}{r}
-C^{t} \\
A
\end{array}\right) \quad \underline{d}=\left(\begin{array}{c}
-C^{M} \\
\underline{b}
\end{array}\right)
$$

Note that $C^{M}$ indicates the worst cost acceptable for the objective function which is determined by the operators according to their experience.

It is observed that we have three types of fuzzy constraints in eqn. 14.

\subsection{Fuzzy constraint related to objective function (generation cost)}

It was mentioned before that the generation cost should be less than $C^{M}$. In addition, a low cost must be given a high membership value. Fig. 2 depicts the membership function $\mu_{\tilde{c}}$ for the fuzzy variable signifying total cost $C$. In this figure, a membership or a satisfaction value of 1 is assigned to any $C$ that is less than $C^{M}-P_{C}$. As $C$ becomes larger than $C^{M}-P_{C}$, the degree of satisfaction will decrease to zero linearly when $C$ is equal to $C^{M}$. The degree of satisfaction is zero for any value of $C$ greater than $C^{M}$. Note that a linear membership function is adopted since we are using the fuzzy LP approach.

The membership function $\mu_{\tilde{c}}$ is expressed as

$$
\mu_{\tilde{c}}= \begin{cases}0 & C \geqslant C^{M} \\ \frac{C^{M}-C}{P_{c}} & C^{M}-P_{c} \leqslant C<C^{M} \\ 1 & C<C^{M}-P_{c}\end{cases}
$$

where $P_{c}=$ cost tolerance value

For a given amount of thermal generation GTHER$M A L$, the total cost $C$ is computed using eqn. 3 . Then the membership function $\mu_{c}$ can be computed using eqn. 16 .

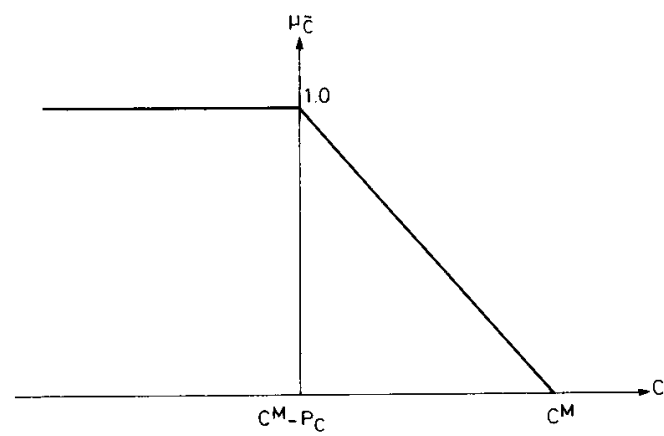

Fig. 2 Membership function $\mu_{c}$ for fuzzy generation cost $C$

\subsection{Fuzzy constraints related to generation-load balance equations}

It is noted from the generation-load balance equations in eqn. 4 that the sum of the generation from thermal units, GTHERMAL $L_{t}$, and the total hydrogenerations $\Sigma P_{i}\left(X_{i t}\right)$ must be equal to the hourly load demand $L_{t}$. In the hydroscheduling problem, $L_{t}$ denotes the hourly load demands in the future. Therefore, $L_{t}$ can only be reached through load forecasting and there are always errors in the forecast hourly loads. As a result, the actual load, $L_{i, ~ a c t u a l}$, can be expressed as the sum of the forecast load, $L_{t, \text { forecast }}$, and the forecast error $\Delta L_{t}$. In other words, we have the following equation

$$
L_{t, \text { actual }}=L_{i, \text { forecast }}+\Delta L_{t}
$$

Note that the forecast load $L_{\text {forecast }}$ is crisp while the forecast error and the actual load are imprecise and are characterised by the fuzzy sets $\Delta \widetilde{L}_{t}$ and $\widetilde{L}_{t, \text { actual }}$. It is also obvious from eqn. 17 that, as long as the membership function for the fuzzy set $\Delta L_{t}$ is known, that for the fuzzy set $L_{\mathrm{z} \text {, actual }}$ can be determined. In the present work, a linearly decreasing membership function in a triangular form is used. The membership value will be 1 for $\Delta l=0$ where no forecast error is observed. For other values of $\Delta l$, the membership function decreases with increasing forecast error. A membership function $\mu_{\tilde{L}}$ as depicted in Fig. 3 is employed. The membership function can be written as follows:

$$
\mu_{\tilde{L}}=\left\{\begin{array}{lc}
\frac{P_{l}-\Delta l}{P_{l}} & 0 \leqslant \Delta l<P_{l} \\
\frac{\Delta l+P_{l}}{P_{l}} & -P_{l}<\Delta l \leqslant 0 \\
0 & \text { otherwise }
\end{array}\right.
$$

where

$P_{L}=$ average forecast error

$\Delta l=$ percentage error in load forecasting

$$
\begin{aligned}
& =\frac{\Delta L_{t}}{L_{t, \text { forecast }}} \times 100 \% \\
& =\frac{L_{t, \text { actual }}-L_{t, \text { forecast }}}{L_{i, \text { forecast }}} \times 100 \%
\end{aligned}
$$


Note that the average forecast error, $P_{l}$, is chosen to be $3 \%$ which is based on the statistics of past forecasting results at the Taiwan Power Company. It is also noted

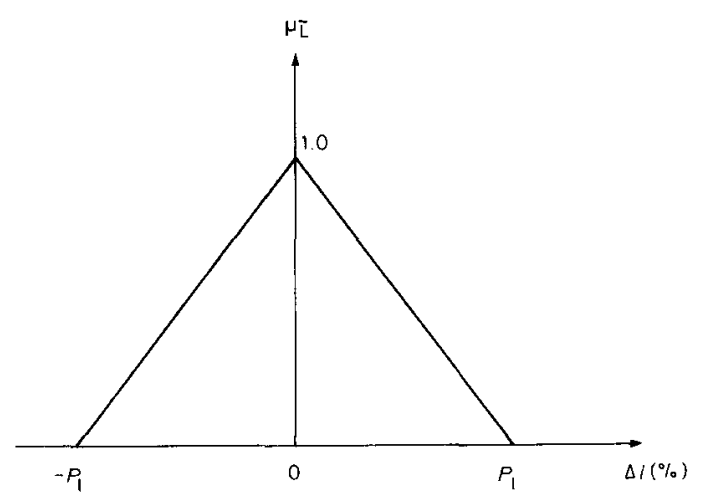

Fig. 3 Membership function $\mu_{t}$ for load demand

that the allowable range for $\Delta l$ is

$$
-P_{l} \leqslant \Delta l \leqslant P_{l}
$$

Therefore, the range for actual hourly loads is

$$
L_{t, \text { forecast }}\left(1-P_{l}\right) \leqslant L_{t, \text { actual }} \leqslant L_{t, \text { forecast }}\left(1+P_{\nu}\right)
$$

For any set of 24 hourly loads $L_{t, \text { actual }}(t=1,2, \ldots, 24)$ within the allowable range specified in eqn. 21 the membership functions $\mu_{\tilde{L}_{t}}(t=1,2, \ldots, 24)$ related to the generation-load balance equations can be computed using eqns. 18 and 19. It is obvious that we have a total of 24 membership functions for the generation-load balance equations.

\subsection{Fuzzy constraints related to water balance equations}

From eqn. 5 , the water balance equation for reservoir $i$ can be rewritten as

$$
\begin{array}{r}
Y_{i t+1}-Y_{i t}-\sum_{j \in N_{i}} X_{j t}+X_{i t}-\sum_{i \in N_{i}} S_{l t}+S_{i t}=R_{i t} \\
i=1,2, \ldots, 10 \quad t=1,2, \ldots, 24
\end{array}
$$

Just as in the case of hourely load demands, there are also errors in the forecast natural inflows $R_{i t}$. Thus, the actual natural inflow $R_{\text {actual }}$, is the sum of the forecast natural inflow, $R_{\text {forecast }}$, and the forecast error, $\Delta R$, that is

$$
R_{\text {actual }}=R_{\text {forecast }}+\Delta R
$$

Based on previous experience with natural inflow forecasting at the Taiwan Power Company, the error is around $15 \%$. Therefore, a membership function $\mu_{\tilde{R}}$ as shown in Fig. 4 is employed for the fuzzy set $\tilde{R}$. The membership function is expressed as follows

$$
\mu_{\tilde{\AA}}= \begin{cases}\frac{P_{r}-\Delta r}{P_{r}} & 0 \leqslant \Delta r<P_{r} \\ \frac{\Delta r+P_{r}}{P_{r}} & -P_{r}<\Delta r \leqslant 0 \\ 0 & \text { otherwise }\end{cases}
$$

IEE Proc.-Gener. Transm. Distrib., Vol. 141, No. 6, November 1994 where

$P_{r}=$ average forecast error

$=15 \%$ in present work

$\Delta r=$ percentage error in natural inflow forecasting

$$
\begin{aligned}
& =\frac{\Delta R}{R_{\text {forecast }}} \times 100 \% \\
& =\frac{R_{\text {actual }}-R_{\text {forecast }}}{R_{\text {forecast }}} \times 100 \%
\end{aligned}
$$

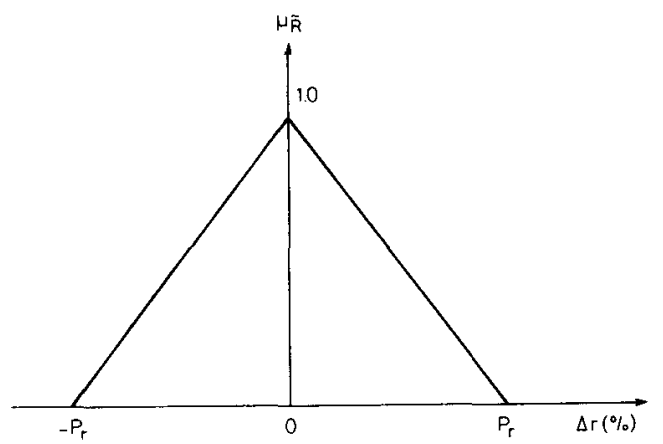

Fig. 4 Membership function $\mu_{R}$ for natural inflow

Note that the membership function $\mu_{\tilde{R}}$ is similar to the membership function $\mu_{\tilde{L}}$ in Fig. 3. Note also that the percentage error is within the range

$$
-P_{r} \leqslant \Delta r \leqslant P_{r}
$$

Therefore, the allowable range for the natural inflow is as follows:

$$
R_{\text {forecast }}\left(1-P_{r}\right) \leqslant R_{\text {actual }} \leqslant R_{\text {forecast }}\left(1+P_{r}\right)
$$

For any natural inflow $R_{i t}(i=1,2, \ldots, 10 ; t=1,2, \ldots$ 24) within the specified range, the membership function $\mu_{\tilde{R}_{14}}$ related to the water balance equation in eqn. 22 can be computed using eqns. 24 and 25 . The number of membership functions for the water balance equations is $10 \times 24=240$.

With the membership functions for the three types of fuzzy constraints in eqn. 14 in hand, we can proceed to determine an 'optimal' solution which best satisfies these constraints.

Based on previous discussions, a solution $X$ to the fuzzy linear programming model is called a feasible solution if $X$ satisfies the crisp constraint, (eqn. 15), and the bounds for hourly loads $L_{\text {, }}$ (t) $(t=1,2, \ldots, 24)$ and the natural inflows $R_{i t}(i=1,2, \ldots, 10 ; t=1,2, \ldots, 24)$ defined in eqns. 21 and 27 , respectively, are satisfied. Among the numerous feasible solutions to the FLP problem, we will choose a solution which meets the fuzzy constraints in eqn. 14 to the highest degree.

It was mentioned that the fuzzy constraints in eqn. 14 can be classified into three types and we can use membership functions $\mu_{\tilde{c}}, \mu_{\tilde{L}}(t=1,2, \ldots, 24)$ and $\mu_{\tilde{R}_{i s}}(i=1,2$, $\ldots, 10 ; t=1,2, \ldots, 24)$ to describe the degrees to which the constraints related to generation cost, generationload balance and water balance, respectively, are satisfied. Since our purpose is to meet all the three types of constraints at the same time, we can use the min-operator to model the intersection of the fuzzy sets. In other 
words, the overall membership function $\mu_{D}(\underline{X})$ for a fuzzy decision $\underline{X}$ is defined as

$$
\begin{aligned}
\mu_{D}(\underline{X})=\min \left[\mu_{C}(\underline{X}), \mu_{L_{i}}(\underline{X}) \quad(t=1,2, \ldots, 24)\right. \\
\left.\mu_{k_{i t}}(\underline{X}),(i=1,2, \ldots, 10 \quad t=1,2, \ldots, 24)\right]
\end{aligned}
$$

A solution $X$ with a high membership value $\mu_{D}(\underline{X})$ is regarded as a good solution which satisfies all the fuzzy constraints to a great extent. Thus the best solution $X^{*}$ to the FLP problem is defined as

$$
\begin{aligned}
& \mu_{\delta}\left(\underline{X^{*}}\right)=\max _{X} \mu_{\bar{D}}(\underline{X}) \\
&=\max _{X} \min \left[\mu_{\mathcal{C}}(\underline{X}), \mu_{L_{i}}(\underline{X}) \quad(t=1,2, \ldots, 24)\right. \\
&\left.\mu_{R_{i}}(\underline{X}) \quad(i=1,2, \ldots, 10 \quad t=1,2, \ldots, 24)\right]
\end{aligned}
$$

If we define $\alpha=\mu_{D}(\underline{X})$ and use $\mu_{i}(\underline{X})$ to denote $\mu_{C}(\underline{X})$, $\mu_{\mathrm{Lt}}(\underline{X})$ and $\mu_{R_{\mathrm{it}}}(\underline{X})$, eqn. 29 can be rewritten as a typical LP formulation.

$$
\max _{X} \alpha=\max _{x} \min _{i=1,2, \ldots .265}\left[\mu_{i}(\underline{X})\right]
$$

subject to

$$
\mu_{i}(\underline{X})-\alpha \geqslant 0 \quad i=1,2, \ldots, 265
$$

$$
0 \leqslant \alpha \leqslant 1 \quad \underline{X} \geqslant 0
$$

Note that $\alpha$ must lie within the range $[0,1]$ because al] membership functions $\mu_{C}(\underline{X}), \mu_{L_{1}}(\underline{X})$ and $\mu_{k_{i}}(\underline{X})$ are within this range.

The optimal solution of eqns. 30 and 31 is the vector $\left(\alpha^{*}, X^{*}\right)$ where $X^{*}$ is the optimal decision and $\alpha^{*}$ is the membership function associated with the decision. The optimal solution can be found by solving one standard (crisp) LP problem. This makes the proposed FLP approach computationally very efficient.

\section{Example}

To demonstrate the effectiveness of the proposed fuzzy linear programming approach, hydroelectric generation scheduling is performed on the Taiwan power system which consists of four Ta-Chia River cascaded plants, three Cho-Shui River plants (including a large pumped storage plant and two cascaded hydroplants) and three hydraulically independent plants. The schematic diagram of the hydroplants along both the Cho-Shui River and the Ta-Chia River is shown in Fig. 5. The hydro system data used for the present work are presented in Table 1. The forecast hourly loads are listed in Table 2 .

The computational results are summarised in Table 3 and Figs. 6 and 7. Table 3 gives the hourly load demands, the total power from the hydrounits and the production costs for the optimal hydrogeneration schedules reached by using the linear programming and the proposed fuzzy linear programming. It is observed from Table 3 that dif-

\section{Table 1: Hydrosystem data}

\begin{tabular}{lcrlcc}
\hline Reservoir & $\underline{Y\left(\mathrm{~km}^{3}\right)}$ & $\bar{\gamma}\left(\mathrm{km}^{3}\right)$ & Plant & $\underline{X}\left(\mathrm{~m}^{3} / \mathrm{s}\right)$ & $\bar{X}\left(\mathrm{~m}^{3} / \mathrm{s}\right)$ \\
\hline Sun-Moon & 13269 & 155685 & Ta-Kuan 2 & -249 & 380 \\
Storage Pond & 1565 & 9407 & Ta-Kuan 1 & 0 & 50 \\
Chu-Kung & 1.6 & 105 & Chu-Kung & 0 & 45 \\
Te-Chi & 89886 & 243120 & Te-Chi & 0 & 217.5 \\
Chin-Shan & 26 & 647 & Chin-Shan & 0 & 174.8 \\
Ku-Kuan & 101 & 6563 & Ku-Kuan & 0 & 133.6 \\
Tien-Lun & 90 & 560 & Tien-Lun & 0 & 68 \\
Li-Wu & 0 & 340 & Li-Wu & 0 & 36.7 \\
Lung-Chien & 0 & 202 & Lung-Chien & 0 & 13.2 \\
I-Hsing & 0 & 1343 & I-Hsing & 0 & 31.7 \\
\hline
\end{tabular}

572 ferent hourly load demands have been assumed in the two approaches. In the LP approach, the forecast hourly loads are directly employed. But these load demands have been modified in the FLP approach by taking the uncertainties in the load demands into account.
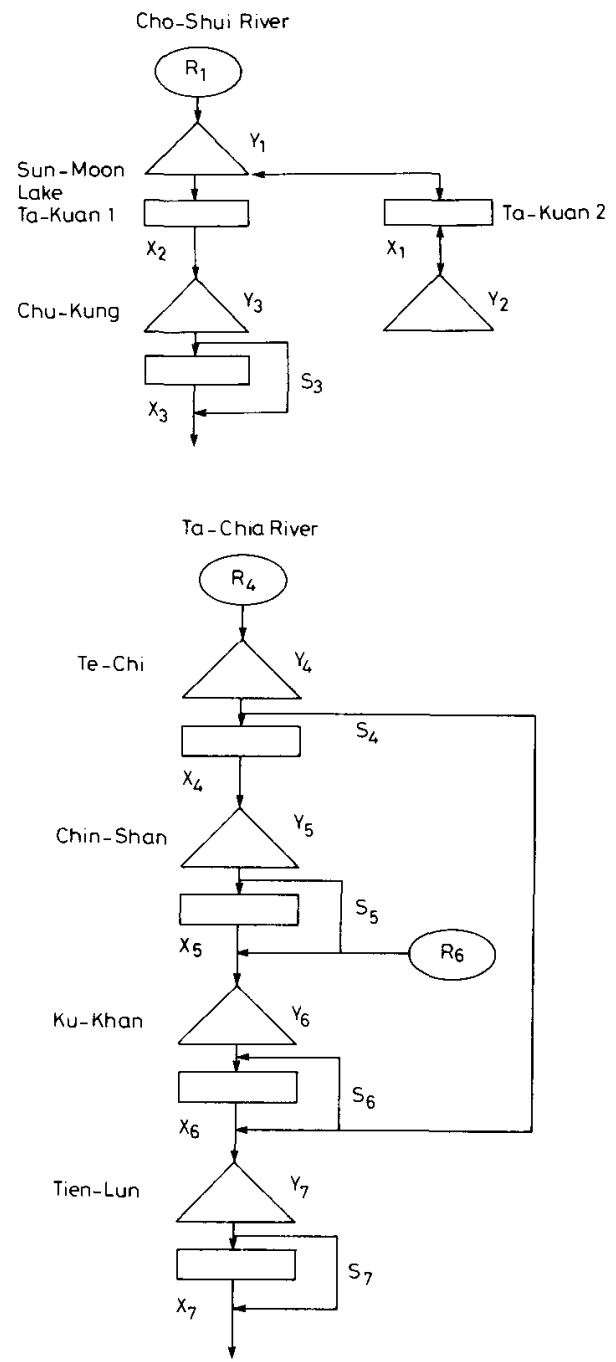

Fig. 5 Schematic diagram of Cho-Shui River and Ta-Chia River $O$ natural inflow

$\triangle$ reservoir

$\Delta$ power house

In most hydrogeneration scheduling studies, a production cost is usually given for the resultant generation schedule. This production cost can only be regarded as a 'predicted' cost which is computed on the assumption that, in actual operation, the hourly loads and hourly inflows will be the same as the forecast loads and the forecast inflows, respectively. Since there are always errors in both forecast loads and inflows, the actual production cost can only be known after the schedule has been executed. Therefore, the production costs given in Table 3 are only the 'predicted' cost under the particular hourly load demands and inflows assumed by each 
Table 2: Forecast hourly loads

\begin{tabular}{lllllllllllllll}
\hline Hour & 1 & 2 & 3 & 4 & 5 & 6 & 7 & 8 & 9 & 10 & 11 & 12 \\
\hline $\begin{array}{l}\text { Load } \\
(\mathrm{MW})\end{array}$ & 7624 & 7340 & 7097 & 6926 & 6818 & 6762 & 6916 & 7467 & 9461 & 10192 & 10531 & 10620 \\
\hline Hour & 13 & 14 & 15 & 16 & 17 & 18 & 19 & 20 & 21 & 22 & 23 & 24 \\
\hline $\begin{array}{l}\text { Load } \\
(\mathrm{MW})\end{array}$ & 9567 & 10744 & 10982 & 10823 & 10560 & 9726 & 9683 & 9897 & 9690 & 9295 & 8968 & 8476 \\
\hline
\end{tabular}

approach. They are, by no means, the 'actual' production costs.

By comparing the total costs from LP and FLP in Table 3, it is observed that the total cost for the generation schedule from the proposed FLP approach is less than that for the generation schedule from conventiona (crisp) LP. The main reason for the difference in the resultant production cost is that the uncertainties in load

Table 3: Results from linear programming (LP) approach and fuzzy linear programming (FLP) approach

\begin{tabular}{lrrrr}
\hline $\begin{array}{l}\text { Hour } \\
h\end{array}$ & \multicolumn{2}{c}{$\begin{array}{c}\text { Load demand, } \\
\text { MW }\end{array}$} & \multicolumn{2}{c}{$\begin{array}{c}\text { Hydroelectric } \\
\text { generation, MW }\end{array}$} \\
\cline { 2 - 5 } & \multicolumn{1}{c}{ LP } & \multicolumn{1}{c}{ FLP } & \multicolumn{1}{c}{ LP } & \multicolumn{1}{c}{ FLP } \\
\hline 1 & 7624 & 7620 & -100 & \multicolumn{1}{c}{-97} \\
2 & 7340 & 7340 & -678 & -678 \\
3 & 7097 & 7096 & -693 & -687 \\
4 & 6926 & 6925 & -750 & -750 \\
5 & 6818 & 6818 & -750 & -750 \\
6 & 6762 & 6761 & -750 & -750 \\
7 & 6916 & 6916 & -750 & -750 \\
8 & 7467 & 7467 & -119 & -119 \\
9 & 9461 & 9431 & 323 & 393 \\
10 & 1019 & 910144 & 789 & 789 \\
11 & 10531 & 10475 & 1469 & 1468 \\
12 & 10620 & 10562 & 1851 & 1851 \\
13 & 9567 & 9541 & 877 & 877 \\
14 & 10744 & 10683 & 1851 & 1851 \\
15 & 10982 & 10922 & 1851 & 1851 \\
16 & 10823 & 10766 & 1851 & 1851 \\
17 & 10560 & 10503 & 1850 & 1851 \\
18 & 9726 & 9689 & 756 & 759 \\
19 & 9683 & 9648 & 630 & 677 \\
20 & 9897 & 9850 & 327 & 334 \\
21 & 9690 & 9648 & 264 & 275 \\
22 & 9295 & 9263 & 203 & 204 \\
23 & 8968 & 8944 & 11 & 22 \\
24 & 8476 & 8465 & -710 & -710 \\
\hline
\end{tabular}

$\alpha=0.951727$

Total cost from $L P=123163560$ NTS $=1.0 \mathrm{pu}$

Total cost from FLP $=122368292$ NT\$ $=0.993543 \mathrm{pu}$

demands and natural inflows are taken into account in the proposed fuzzy linear programming approach. In the process of reaching an 'optimal' hourly generation schedule, we try to reduce the production cost by keeping the membership function for the cost $\mu_{c}$ as high as possible. Therefore, the reduction in production cost is achieved by taking advantage of the uncertainties in load demands and natural inflows. This observation is confirmed by the different hourly loads and hourly hydrogeneration in Table 3.

Figs. 6 and 7 illustrate the water release schedules for two of the ten reservoirs over the $24 \mathrm{~h}$ scheduling period. Details of the water release for other reservoirs are not given due to limited space. It is observed from Fig. 6 that different water release schedules have been obtained by using the two approaches since different hourly inflows are assumed by the two approaches. In the LP approach,

IEE Proc.-Gener. Transm. Distrib., Vol. 14I, No. 6, November 1994 the forecast hourly inflows are directly employed. But these natural inflows have been modified in the FLP approach by taking the uncertainties in the natural inflows into account.

Since the membership functions $\mu_{\bar{C}}, \mu_{\bar{L}}$ and $\mu_{\bar{R}}$ described in Figs. 2-4, respectively, have been chosen somewhat arbitrarily, it is desirable to see how different parameters $P_{C}, P_{l}$ and $P_{r}$ in the membership functions will affect the final result. The total costs and alpha value of the optimal hydro generating schedules obtained by using different parameters of $\mu_{\bar{C}}, \mu_{\mathrm{t}}$ and $\mu_{\bar{\kappa}}$ are compared in Table 4

From the results in Table 4, the following observations can be made:

(i) By comparing the total costs of the optimal schedules obtained by using the same parameters of the membership function $\mu_{L}$ and $\mu_{R}$ for the load demand error

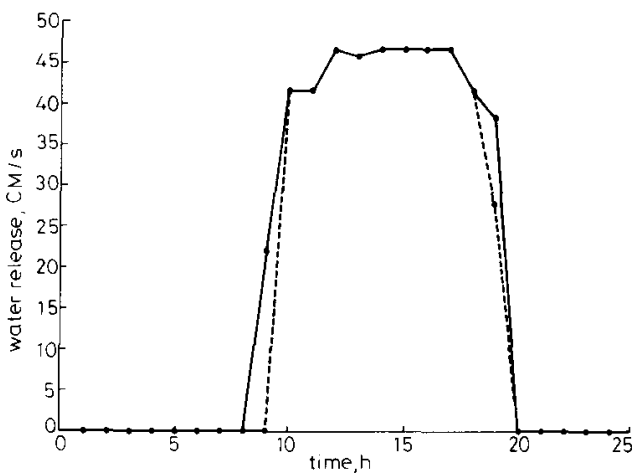

Fig. 6 Water release for Ta-Kuan I hydroplant from $L P$ and FLP -O- LP

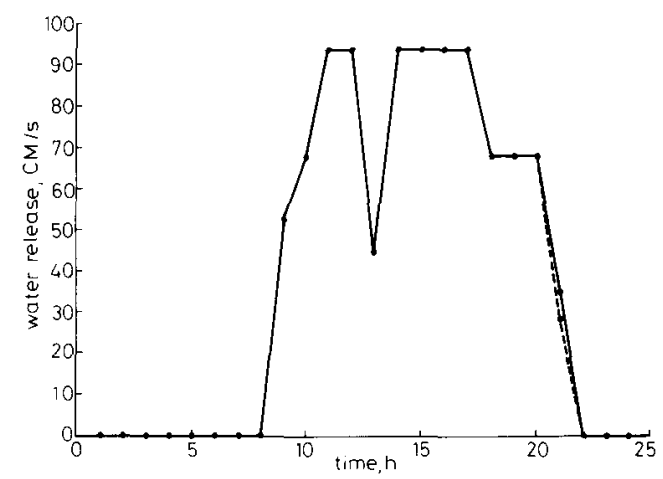

Fig. 7 Water release for Ku-Kuan hydroplant from $L P$ and $F L P$ $\begin{array}{ll}-\supset- & \text { LP } \\ -x & \text { FLP }\end{array}$ 
Table 4: Summary of total costs and $\alpha$ for different $\mu_{C}, \mu_{L}$ and $\mu_{K}$

\begin{tabular}{|c|c|c|c|c|c|c|c|c|}
\hline \multirow{3}{*}{$\begin{array}{l}\text { Cost tolerance } \\
\text { value } P_{c}(\mathrm{pu})\end{array}$} & \multicolumn{4}{|c|}{$P_{r}=15 \%$} & \multicolumn{4}{|c|}{$P_{t}=3 \%$} \\
\hline & \multicolumn{2}{|c|}{$P_{i}=3 \%$} & \multicolumn{2}{|c|}{$P_{1}=2 \%$} & \multicolumn{2}{|c|}{$P_{r}=0 \%$} & \multicolumn{2}{|c|}{$P_{r}=20 \%$} \\
\hline & $\alpha$ & $\mathrm{TC}(\mathrm{pu})$ & $\alpha$ & $\mathrm{TC}(\mathrm{pu})$ & $\alpha$ & TC (pu) & $\alpha$ & TC (pu) \\
\hline $\begin{array}{l}0.008 \\
0.009 * \\
0.01\end{array}$ & $\begin{array}{l}0.999429 \\
0.951727^{*} \\
0.908366\end{array}$ & $\begin{array}{l}0.994067 \\
0.993543^{*} \\
0.993067\end{array}$ & $\begin{array}{l}0.895618 \\
0.852522 \\
0.813372\end{array}$ & $\begin{array}{l}0.994683 \\
0.994216 \\
0.993792\end{array}$ & $\begin{array}{l}0.999275 \\
0.939538 \\
0.886539\end{array}$ & $\begin{array}{l}0.994068 \\
0.993626 \\
0.993233\end{array}$ & $\begin{array}{l}0.999529 \\
0.959826 \\
0.923136\end{array}$ & $\begin{array}{l}0.994066 \\
0.993488 \\
0.992954\end{array}$ \\
\hline
\end{tabular}

$\mathrm{TC}=$ total cost

* Case in above example.

and natural inflow error, but using different tolerance values $\boldsymbol{P}_{\boldsymbol{C}}$ for the membership function $\mu_{C}$, it is observed that the total cost can be reduced by increasing the tolerance values $\boldsymbol{P}_{C}$ for $\mu_{C}$. This is as expected since a greater tolerance value for $\mu_{C}$ implies a lesser penalty for the decrease in the total cost. Therefore, the FLP will try to find a hydrogenerating schedule which has a lower cost if a larger tolerance value is employed for $\mu_{\tilde{c}}$. It is also observed from Table 4 that the optimal decision will have a smaller $\alpha$ if a greater tolerance value $P_{C}$ is employed. In summary, the total cost of the optimal hydrogenerating schedule can be reduced by using a great tolerance value at the price of having a lower $\alpha$. In practical applications, a decision with too low an $\alpha$ is unacceptable since a small $\alpha$ implies that both the load and the inflow level used are far from the actual load and inflow.

(ii) Under a forecast load error of 3\%, the total cost of the optimal schedule can be reduced by using a great natural inflow tolerance error. It is also observed that, under a forecast inflow error of $15 \%$, the total cost of the optimal schedule can be increased by using a small load demand tolerance error. This is as expected since the economic factor is considered.

\section{Conclusions}

A novel technique using fuzzy linear programming has been developed for the short-term hydroelectric generation scheduling of a power system. A standard LP algorithm can be applied to the fuzzy formulation. To take the errors in both forecast hourly loads and inflows into account, membership functions are derived for the total cost, the load demand and the natural inflow using fuzzy set notations. With these membership functions at hand, an algorithm for fuzzy linear programming is presented to reach the optimal hydrogenerating schedule under the fuzzy environment. The developed algorithm is applied to the hydroelectric generation scheduling of the Taiwan power system which consists of 10 hydroplants. Results reveal that the proposed fuzzy linear programming is very effective in reaching an optimal hydrogenerating schedule when the imprecision in the hourly loads and inflows is considered. The effect of different membership functions on the final result is also examined. It is obvious that the proposed fuzzy linear programming approach requires more computer time than the linear programming method. The required CPU time for the
FLP on a Sun Workstation is $511 \mathrm{~s}$, whilst that for LP is $251 \mathrm{~s}$ on the same machine.

\section{References}

1 CARVALHO, M.F., and SOARES, S.: 'An efficient hydro-therma scheduling algorithm', IE EE Trans., 1987, PWRS-4, pp. 537-542

2 SANDELL, N.R., BERTSEKAS, D.P., SHAW, J.J., GULLY, S.W and GENDRON, R.: 'Optimal scheduling of large-scale hydrothermal power systems'. Proc. IEEE International Large-scale Systems Symposium, 1982, pp. 141-147

3 HABIBOLLAZADEH, H., and BUBENKO, J.A.: 'Application of decomposition techniques to short-term operation planning of hydro-thermal power system', IEEE Trans., 1986, PWRS-1, pp. 44

4 SHERKAT, VR MOSLEHI $K$, LO, EO, SANCHEZ, $G$, and DIAZ, J.: 'Modular and flexible software for medium- and shortterm hydro-thermal scheduling', IEEE Trans, 1988, PWRS-3, pp. $1390-1396$

5 HEINSSON, E.B.: 'Optimal short-term operation of a purely hydroelectric system', IEEE Trans., 1988, PWRS-3, pp. 1072-1077

6 AMADO, S.M., and RIBEIRO, C.C.: 'Short-tern generation scheduling of hydraulic multi-reservoir multi-area interconnected Uling ', hEE Trans 1987, PWRS-2, pp 758-763

7 WAIGHT, J.G., ALBUYEH, F., and BOSE, A.: 'Scheduling of genWAIGHT, J.G., ALBUYEH, F., and BOSE, A.: 'Scheduling of genIEEE Trans., 1981, PAS-100, pp. 2226-2230

8 TRISTAO, A., MARIO, V.F., and JERSON, K.: 'A risk-constrained stochastic dynamic programming approach to the operation planning of hydrothermal systems', IEEE Trans., 1985, PAS-104, pp. $273-279$

9 WOOD, A.J., and WOLLENBERG, B.F.: 'Power generation operation and control' (Wiley, New York, 1984)

10 ZADEH, L.A.: 'Fuzzy sets', Information and Control, 1965, 8, pp. 338-353

11 ZIMMERMANN, H.J.: 'Fuzzy set theory and its applications' (Kluwer-Nijhoff Publishing, 1985)

12 SU, C.C., and HSU, Y.Y.: 'Fuzzy dynamic programming: an application to unit commitment', IEEE Trans., 1991, PWRS-6, pp. 12311237

13 HSU, Y.Y., and KUO, H.C.: 'Fuzzy-set based contingency ranking', IEEE Trans., 1992, PWRS-7, pp. 1189-1196

14 HSU, Y.Y., and HO, K.L.: 'Fuzzy expert systems: an application to short-term load forecasting', IEE Proc. C, 1992, 139, (6), pp. 471477

15 ECONOMAKES, E.: 'Application of fuzzy concepts to power demand forecasting', IEEE Trans., 1979, SMC-9, pp. 651-657

16 TOMSOVIC, $K$.: 'A fuzzy linear programming approach to the reactive power/voltage control problem'. IEEE 1991 Summer Meeting, Paper 91 SM 435-8 PWRS

17 ABDUL-RAHMAN, K.H., and SHAHIDEHPOUR, S.M.: 'A fuzzy-based optimal reactive power control'. IEEE 1992 Summer Meeting Paper 92 SM 402-8 PWRS

18 MIRANDA, V., and SARAIVA, J.T.: 'Fuzzy modelling of power system optimal load flow', IEEE Trans., 1992, PWRS-7, pp. 843849 